\title{
EDITORIAL
}

\section{DOCTOR OF PHILOSOPHY - A NEW HORIZON TOWARDS ADVANCEMENT IN PHYSICAL THERAPY}

Physical therapy is on the rise as it is among the flourishing disciplines of medical science and is an integral part of a Rehabilitation team. It is an independent health care profession that deals in identifying and optimizing quality of life within the spheres of promotion, prevention, treatment and restoration. The need of Physical Therapy education in Pakistan was acknowledged back in 1950's, when a 2 years diploma was started which over the years advanced into a Bachelor's degree. Later, this degree was upgraded to 5 years Doctor of Physical Therapy in order to meet the International standard following the "Vision 2020 of American Physical Therapy Association".

Training and education is a continuous process that may be measured by level of knowledge, skills or aptitude. The changes in the field of medical science and technology will require a physiotherapist to be occupied in any form of continuous learning even if they have reached a mastery level. The constant development and advancement of tools, equipment, therapeutic skills and techniques will continue to grow worldwide. There are many ways to include a component of continuous learning in your training and education plan. It is a well acknowledged fact that the pursuit of knowledge through research leads to a well-educated and intellectual society, a highly trained professional team and at the same time it also contributes significantly in creating Academic Leaders. Doctor of Philosophy (PhD) program provides research training that promotes the maturity of independent research skills of professionals. These skills include the ability to define a significant problem, to build up command of appropriate conceptual ideas and to relate the research topic in a broader spectrum of knowledge in the relevant disciplinary area.

During the 60 years of existence, the field of physical therapy in Pakistan is still behind the international level of competence especially in terms of evidence based practice and research skills. This level can only be attained by getting the highest degree of academic qualification in this field i.e. PhD in Physiotherapy. Fortunately there are a few Universities in Pakistan that recently started offering the PhD program and many others are in process to launch this Program but at the same time the quality and standard of their research skills, core areas of teaching and specialization in evidence based practice is debatable. Evidence-based practice is gradually being recognized as a priority by all professional physiotherapy organization, leading researchers and clinicians worldwide. According to the predictions of the Bureau of Labor Statistics the opportunities of employment are expected to grow $39 \%$ for careers that require a masters' degree or higher and fortunately physical therapy has been ranked 6th on their list of the fastest growing jobs.

The emergence of a PhD program in physical therapy has also opened new doors for the advancement of this field in Pakistan. Our Physiotherapists are well aware of the fact that clinical research in the field of physiotherapy is very important for the profession just like other medical fields. Even in Pakistan, there is a small group of qualified Physiotherapists who are now using what is called evidence-based therapy practice as the source for planning their treatments that are a combination of scientific research, clinical reasoning and the individual's goals and needs. Unfortunately, a small group will not be effective in implementing these skills throughout the nation. It is extremely crucial to train the rest of the colleague's in order to secure clinical practice as well developing academic leaders.

Holders of the PhD will be able to teach at the university level as assistant, associate or professors. There was a time when the PhD degree was necessary to begin a career in academia; though not entirely true today, the degree is still generally a guarantee of an academic position at universities worldwide. Furthermore, your expertise in the field also gives you the opportunity to acquire senior positions in clinical settings. Every research conducted by a therapist helps the entire fraternity to gain benefit and apply better skills and more effective techniques in their practice. Unfortunately, due to the absence of a regulatory body in Pakistan, the quality of education and research at institutions has brought a major decline in the progression of physiotherapy. At the same time there are Universities that are setting a standard in the field of physical therapy by providing high-quality education, clinical exposure and professional grooming.

Every physiotherapist who has a vision and has set goals to prosper in this field will urge for the establishment of the PhD program in Physical Therapy. This degree will signify that they have attained the greatest level of competence in their field of study. It will also be a signal to the employers in both clinical and academic systems that they have the requisite analytical skills to carry out rigorous research tasks and train others in methodology and statistics. I believe ensuring quality higher education is one of the most important things we can do for the future of Physical Therapy. Education is definitely an ongoing process and as Henry Ford once said, "Anyone who keeps learning stays young." I hope we all remain indulged in this learning process and keep our minds young with advance physiotherapy skills and knowledge.

\section{Dr. Nabiha Ahmed}

Senior Lecturer / Assistant Manager

Physiotherapy Department

Ziauddin Hospital Clifton 